\title{
Hospital Variation in Cholecystectomies in The Netherlands: A Nationwide Observational Study
}

\author{
Carmen S.S. Latenstein $^{\mathrm{a}}$ Sarah Z. Wennmacker ${ }^{\mathrm{a}}$ Stef Groenewoud ${ }^{\mathrm{b}}$ \\ Mark W. Noordenbos ${ }^{b}$ Femke Atsma ${ }^{b}$ Philip R. de Reuver ${ }^{a}$

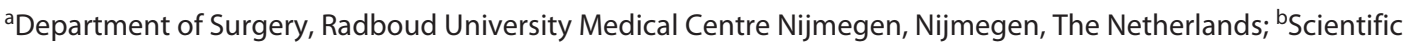 \\ Institute for Quality of Healthcare (IQ healthcare), Radboud University Medical Centre, Nijmegen, The Netherlands
}

\section{Keywords}

Hospital variation - Cholecystectomy · Contributing factors . Clinical outcome

\begin{abstract}
Background: Practice variation generally raises concerns about the quality of care. This study determined the longitudinal degree of hospital variation in proportion of patients with gallstone disease undergoing cholecystectomy, while adjusted for case-mix, and the effect on clinical outcomes. Methods: A nationwide, longitudinal, database study was performed in all hospitals in the Netherlands in 2013-2015. Patients with gallstone disease were collected from the diagnosis-related group database. Hospital variation in casemix-adjusted cholecystectomy rates was calculated per year. Clinical outcomes after cholecystectomy were compared between hospitals in the lowest/highest 20th percentile of the distribution of adjusted cholecystectomy rates in all 3 subsequent years. Results: In total, 96,673 patients with gallstones were included. The cholecystectomy rate was $73.6 \%$. In 2013-2015, the case-mix-adjusted performance of cholecystectomies was in hospitals with high rates 1.5-1.6 times higher than in hospitals with low rates. Hospitals with a high adjusted cholecystectomy rate had a higher laparoscopy rate, shorter time to surgery, and less emergency depart-
\end{abstract}

karger@karger.com www.karger.com/dsu

Karger"

BOPEN ACCESS
(C) 2020 The Author(s)

Published by S. Karger AG, Basel

This article is licensed under the Creative Commons AttributionNonCommercial-NoDerivatives 4.0 International License (CC BYNC-ND) (http://www.karger.com/Services/OpenAccessLicense). Usage and distribution for commercial purposes as well as any distribution of modified material requires written permission. ment visits after a cholecystectomy compared to hospitals with a low-adjusted cholecystectomy rate. Conclusion: Hospital variation in cholecystectomies in the Netherlands is modest, cholecystectomy rates varies by $<2$-fold, and variation is stable over time. Cholecystectomies in hospitals with high adjusted cholecystectomy rates are associated with improved outcomes.

(c) 2020 The Author(s)

Published by S. Karger AG, Basel

\section{Introduction}

Cholecystectomy is the most commonly performed gastrointestinal surgical procedure worldwide, accounting for over 1,500,000 surgeries in the USA and almost 25,000 surgeries in the Netherlands, every year $[1,2]$. A cholecystectomy is mostly an elective straightforward operation while watchful waiting is an accepted alternative in selected patients [3]. The proportion of cholecystectomies has increased over the past decades, although the

Previous presentation: This research was presented at the Academic Surgical Congress - February 7, 2019, a congress of the Dutch Society of Surgeons - May 16, 2019, and the 13th Congress of the EuropeanAfrican Hepato-Pancreato-Biliary Association (E-AHPBA 2019), June $5,2019$. 
indication for surgery was not broadened according to guidelines [4-6]. Because watchful waiting is an alternative for a cholecystectomy, it is often regarded as the textbook example of practice variation in gastrointestinal surgery.

The United States' Dartmouth Atlas of Healthcare and several European reports show that whether a patient undergoes a general surgical procedure or not heavily depends on the region or hospital [7-9]. The Dartmouth Atlas of Healthcare shows that in some regions in the USA, the cholecystectomy rate is 4 times higher than that in other regions. In 2011, hospital variation in cholecystectomies was researched in the Netherlands and showed that it varies by 5 -fold [10]. This report gave a persistent negative connotation to this type of surgery, but additional research into the contribution to this variation was not conducted. The hypothesis is that hospital variation is reduced since 2011 and that hospital characteristics may contribute to hospital variation.

This study aimed to determine longitudinal hospital variation in cholecystectomy rates in patients with gallstone disease in a nationwide cohort in the Netherlands, while adjusting for case-mix. Second, the clinical outcomes and hospital characteristics were compared between those having low/high case-mix-adjusted cholecystectomy rates.

\section{Methods}

\section{Study Population and Data}

For the present study, data of patients with gallstone disease from 2013, 2014, and 2015 were extracted from a routinely collected nationwide database with hospital data. In the Netherlands, hospital care reimbursements are based on diagnosis-related groups (DRGs). These DRGs consist of an average of healthcare costs for a combination of various treatments. These do not give information about the actual care provided. The database included both DRGs as well as the healthcare activities, so the actual provided care was used in the analyses [2]. These healthcare activities are registered by all Dutch hospitals. At time of the study, the coverage was $90 \%$ for $2013,90 \%$ for 2014 , and $80 \%$ for 2015 (due to administrative delays in registries).

All newly diagnosed gallstone patients (cholecystolithiasis, Dutch code: 0303-323) in each year were selected from the database based on diagnosis codes. This mainly includes patients who present at the surgical outpatient clinic but also inpatient hospital episodes. Diagnosis codes did not differentiate between complicated (e.g., cholecystitis) and uncomplicated gallstone disease.

This research was performed in accordance with the ethical standards of the Helsinki Declaration of 1975. The Strengthening the Reporting of Observational studies in Epidemiology guideline and the REporting of studies Conducted using Observational Routinelycollected health Data (RECORD) statement were followed $[11,12]$.

\section{Data Collection}

In the database, detailed information about actual performed healthcare procedures was available. Patients with gallstone disease and the following healthcare procedure codes were assigned to the cholecystectomy group: laparoscopic cholecystectomy, cholecystectomy, laparoscopic cholecystectomy including choledochotomy, cholecystectomy including choledochotomy, laparoscopic cholecystectomy and appendectomy, and cholecystectomy and appendectomy. Patients with gallstones without surgical treatment were assigned to the conservative treatment group. Conservatively treated patients were assigned to the last visiting hospital. Patients who received surgery were assigned to the hospital where the surgery took place.

Patient characteristics and type of treatment included sex, age, socioeconomic status (SES) score [13], time interval between diagnosis and surgery, and type of surgery (open or laparoscopically). Clinical outcomes included emergency department visits within 30 days after diagnosis. In patients who underwent surgery, additional data on emergency department visits, readmissions, reoperations, and the postoperative performance of an endoscopic retrograde cholangiopancreatography, all within 30 days of operation, were assessed.

Type of hospital (general, academic, and private) was also available in the database. Hospital characteristics, for the entire hospital and more specific for the department of surgery, were collected from the register of the Dutch Ministry of Healthcare, Welfare and Sports summarizing hospital characteristics from $2014[14,15]$. The present study did not require approval from an ethics committee in the Netherlands. The DRG database (with both DRGs as well as the healthcare activities) did not require written informed consent form patients.

\section{Study Outcomes}

The cholecystectomy rate was defined as the proportion of patients with gallstone disease undergoing cholecystectomy. The primary outcome of the study was the variation in cholecystectomy rates between hospitals. The cholecystectomy rate was adjusted for case-mix (sex, age, and SES score). First, the variation in case-mixadjusted cholecystectomy rate per 1,000 patients was calculated between all Dutch hospitals. Academic hospitals and private clinics were excluded in further analysis. Second, the variation in casemix-adjusted cholecystectomy rate per 1,000 patients was calculated between general hospitals only. This second analysis will provide more information about variation between hospitals with similar patient populations and about the contribution of type of hospital to hospital variation. Third, clinical outcomes between hospitals with a low and high case-mix-adjusted cholecystectomy rate were compared. Finally, hospital characteristics were compared between hospitals with a low and high case-mix-adjusted cholecystectomy rate.

\section{Statistical Analysis}

Descriptives

Patient characteristics were calculated for all individual patients with gallstone disease expressed per year. Age was presented as mean with standard deviation and sex and surgical treatment were presented as percentages. 
Hospital Variation

Hospital variation was analyzed between all types of hospitals and only between general hospitals. All analyses were based on individual patient data and not on population-based data. To avoid small sample variation, hospitals with $<30$ patients with gallstones in a year and those that performed $<5$ surgical operations of interest per year were excluded from analysis.

First, the case-mix-adjusted cholecystectomy rate per hospital was calculated. The calculation of the case-mix-adjusted cholecystectomies per 1,000 gallstone patients consisted of 3 steps: first, a logistic regression with surgery/conservative treatment as outcome and age, sex, and SES as covariates was performed to assess the expected cholecystectomies per hospital; second, expected and performed cholecystectomies were standardized to cholecystectomies per 1,000 gallstone patients per hospital; third, the performed cholecystectomies per 1,000 gallstone patients were divided by the expected cholecystectomies per 1,000 gallstone patients, then this ratio was multiplied by the national average of cholecystectomies.

Second, to express the amount of variation, 2 types of factor scores were calculated $[16,17]$. The factor score is a measure to describe the degree of hospital variation. It shows by which factor the highest scores differ from the lowest scores. The factor score was calculated by dividing the mean of the case-mix-adjusted cholecystectomy rates of the 3 hospitals with the highest adjusted rates by the mean of the case-mix-adjusted cholecystectomy rate of the 3 hospitals with the lowest adjusted rates. Additionally, to exclude the influence of outliers, factor scores were based on the 95th and 5 th percentile of the distribution of case-mix-adjusted cholecystectomy rates. The case-mix-adjusted cholecystectomy rate of the 95th percentile was divided by the adjusted rate in the 5 th percentile. International literature showed that a factor score below 2.0 is modest [18]. This means that a patient visiting the hospital in the 95th percentile has a 2 times higher chance of undergoing treatment than a hospital in the 5 th percentile. Factor scores were calculated between all hospitals and between general hospitals only. Hospital variation was presented in bar charts.

\section{Comparing Hospitals with Low and High Adjusted}

Cholecystectomy Rates

Academic hospitals and private clinics were excluded to compare clinical outcomes and hospital characteristics between general hospitals with a low or high case-mix-adjusted cholecystectomy rate. A hospital was defined as "hospital with a low adjusted cholecystectomy rate" when a hospital appeared in the lowest 20th percentile of the distribution of the case-mix-adjusted cholecystectomy rates in all 3 subsequent years $(2013,2014$, and 2015). A hospital was defined as "hospital with a high adjusted cholecystectomy rate" when a hospital appeared in the highest 20 th percentile of the distribution of the casemix-adjusted cholecystectomy rate in all 3 subsequent years.

Days to operation were presented in means with standard deviation. Other patient- and treatment characteristics and clinical outcomes were presented as percentages. Hospital characteristics were presented as means per hospital and percentages, respectively, for hospitals with a low adjusted cholecystectomy rate and hospitals with a high adjusted cholecystectomy rate. Analysis of continuous data was done using Student's $t$ test for normally distributed data and Mann-Whitney U test for skewed data. Dichotomous data were analyzed using $\chi^{2}$ test. Associations with a $p$ value $<0.05$ will be considered statistically significant. Analyses were performed with $\mathrm{R}$ version 3.5.1 and SPSS version 22.
Table 1. Patient characteristics and hospital variation factor scores

\begin{tabular}{|c|c|c|}
\hline & $\begin{array}{l}\text { All } \\
\text { hospitals }\end{array}$ & $\begin{array}{l}\text { General } \\
\text { hospitals }\end{array}$ \\
\hline \multicolumn{3}{|l|}{2013} \\
\hline Hospitals, $n$ & 83 & 74 \\
\hline Patients, $n$ & 32,499 & 30,759 \\
\hline Sex, $n(\% \mathrm{M})$ & $10,556(32.5)$ & $9,886(32.1)$ \\
\hline Age, mean (SD) & $53.8(16.7)$ & $53.8(16.7)$ \\
\hline Surgical treatment, $n(\%)$ & $24,041(74.0)$ & $22,927(74.5)$ \\
\hline Factor score ${ }^{\mathrm{a}}$ & $1.47(2.03)$ & $1.38(1.92)$ \\
\hline \multicolumn{3}{|l|}{2014} \\
\hline Hospitals, $n$ & 77 & 68 \\
\hline Patients, $n$ & 33,723 & 31,987 \\
\hline Sex, $n(\% \mathrm{M})$ & $11,275(33.4)$ & $10,561(33.0)$ \\
\hline Age, mean (SD) & $54.2(16.6)$ & $54.1(16.6)$ \\
\hline Surgical treatment, $n(\%)$ & $24,702(73.2)$ & $23,611(73.8)$ \\
\hline Factor score ${ }^{\mathrm{a}}$ & $1.57(2.02)$ & $1.28(1.48)$ \\
\hline \multicolumn{3}{|l|}{2015} \\
\hline Hospitals, $n$ & 68 & 59 \\
\hline Patients, $n$ & 30,451 & 28,804 \\
\hline Sex, $n(\% \mathrm{M})$ & $10,186(33.5)$ & $9,560(33.2)$ \\
\hline Age, mean (SD) & $54.5(16.7)$ & $54.4(16.7)$ \\
\hline Surgical treatment, $n(\%)$ & $21,887(71.9)$ & $20,865(72.4)$ \\
\hline Factor score ${ }^{a}$ & $1.56(1.97)$ & $1.27(1.37)$ \\
\hline
\end{tabular}

The factor score is a measure to describe the degree of hospital variation. It shows by which factor the highest scores differ from the lowest scores. Factor scores were based on the 95th and 5th percentile of the distribution of case-mix-adjusted cholecystectomy rates. The case-mix-adjusted cholecystectomy rate of the 95th percentile was divided by the case-mix-adjusted cholecystectomy rate in the 5th percentile. Additionally, we calculated the factor scorebydividingthemean ofthecase-mix-adjusted cholecystectomy rates of the 3 hospitals with the highest adjusted rates by the mean of the 3 hospitals with the lowest adjusted rates. The number of hospitals went down each year due to fusion of hospitals. At time of the study, the coverage was $90 \%$ for $2013,90 \%$ for 2014 , and $80 \%$ for 2015 (due to administrative delays in registries). Therefore, the number of patients is lower in 2015. a $95 \mathrm{th} / 5$ th percentile (top3/ bottom3).

\section{Results}

\section{Patient Population}

In 2013, 2014, and 2015, respectively, 32,499; 33,723; and 30,451 were diagnosed with gallstone disease. Three, 5 , and 4 hospitals were excluded due to low patient or operation numbers, respectively, in 2013, 2014, and 2015. The patient characteristics are summarized in Table 1.

\section{Variation between All Hospitals}

For 2014, the case-mix-adjusted cholecystectomies per 1,000 gallstone patients per hospital are illustrated in Fig- 


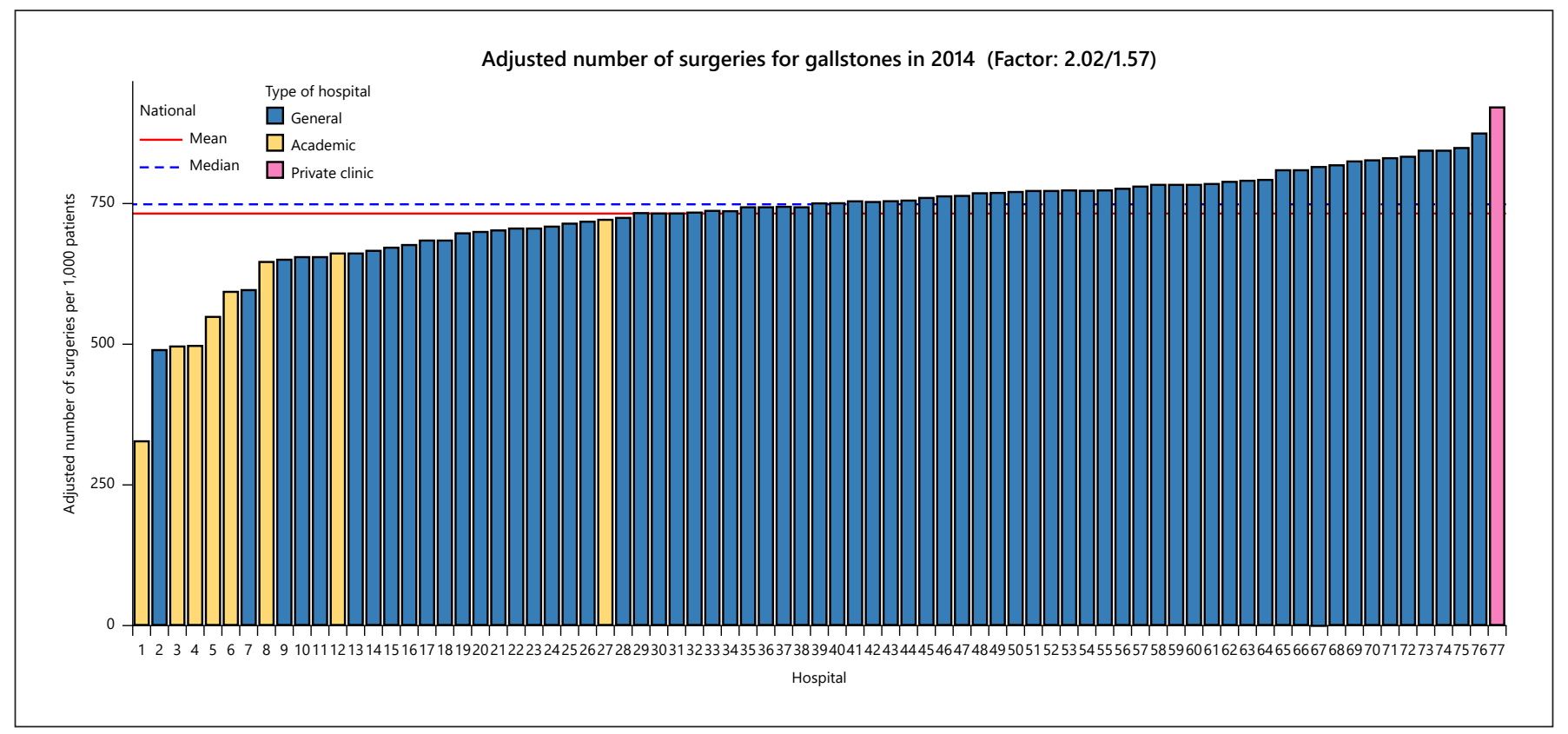

Fig. 1. Hospital variation in cholecystectomies in all Dutch hospitals. Case-mix-adjusted cholecystectomies per 1,000 patients per hospital. Adjustments were made for sex, age, and social-economic status. Horizontal lines illustrate the mean/median case-mix-adjusted cholecystectomies per 1,000 patients. Each bar represents 1 hospital. Yellow bars implicate academic hospitals, pink bars implicate private clinics, and blue bars implicate general hospitals.

Table 2. Difference in treatment characteristics and clinical outcome between hospitals with low and high adjusted cholecystectomy rates

\begin{tabular}{|c|c|c|c|}
\hline \multirow[t]{2}{*}{ Clinical outcome } & \multicolumn{2}{|l|}{ Hospital } & \multirow[t]{2}{*}{$p$ value } \\
\hline & Low $^{\mathrm{a}}$ & High $^{b}$ & \\
\hline Patients, $n$ & 1,938 & 1,770 & \\
\hline Sex, $n(\% \mathrm{M})$ & 627 (32.4) & 547 (30.9) & \\
\hline Age, mean (SD) & $52.84(1.3)$ & $53.74(2.7)$ & \\
\hline Conservative treatment & $n=677$ & $n=305$ & \\
\hline Emergency department visit $<30$ days after diagnosis, $n(\%)$ & $50(7.4)$ & $33(10.8)$ & 0.073 \\
\hline Operative treatment & $n=1,261$ & $n=1,465$ & \\
\hline Laparoscopic operation, $n(\%)$ & $1,185(94.0)$ & $1,430(97.6)$ & $<0.001$ \\
\hline Days to surgery, weighted mean (SD) & $47.3(11.4)$ & $27.3(4.5)$ & 0.009 \\
\hline Emergency department visit $<30$ days after diagnosis, $n(\%)$ & $43(3.4)$ & $43(2.9)$ & 0.479 \\
\hline Emergency department visits $<30$ days after surgery, $n(\%)$ & $112(8.9)$ & $74(5.1)$ & $<0.001$ \\
\hline Readmission $<30$ days after surgery, $n(\%)$ & $47(3.7)$ & $39(2.7)$ & 0.113 \\
\hline Reoperation $<30$ days after surgery, $n(\%)$ & $8(0.63)$ & $12(0.82)$ & 0.573 \\
\hline ERCP $<30$ days, $n(\%)$ & $3(0.24)$ & $4(0.27)$ & 0.857 \\
\hline
\end{tabular}

Academic hospitals and private clinics were excluded to compare clinical outcomes between general hospitals withaloworhighcase-mix-adjustedcholecystectomyrate.ERCP,endoscopicretrogradecholangiopancreatography. a A hospital was defined as "hospital with a low adjusted cholecystectomy rate" when a hospital appeared in the lowest 20th percentile of the distribution of the case-mix-adjusted cholecystectomy rates in all 3 subsequent years $\left(2013,2014\right.$, and 2015). ${ }^{\text {b }}$ A hospital was defined as "hospital with a high adjusted cholecystectomy rate" when a hospital appeared in the highest 20th percentile of the distribution of the case-mix-adjusted cholecystectomy rate in all 3 subsequent years. 
Table 3. Difference in hospital characteristics between hospitals with low and high adjusted cholecystectomy rates

\begin{tabular}{llll}
\hline Hospital characteristic & \multicolumn{2}{l}{ Hospital } & \multirow{2}{*}{ p value } \\
\cline { 2 - 3 } & Low $^{\mathrm{a}}$ & High $^{\mathrm{b}}$ & \\
\hline Surgical teaching hospital & & \\
Another hospital, same city - yes/no & $4 / 1$ & $1 / 4$ & \\
Characteristics per hospital & $3 / 2$ & $1 / 4$ & \\
Annual revenue, mean - million & & & 0.424 \\
Newly registered medical conditions, mean & 190 & 133 & 0.563 \\
$\quad$ Fixed price, \% & 172,640 & 141,779 & $<0.001$ \\
$\quad$ Not fixed/free price, \% & 82.3 & 6.5 & 0.337 \\
Operation rooms, mean & 11 & 7 & 0.641 \\
Bed capacity, mean & 399 & 334 & 0.609 \\
Staff, mean & 2,128 & 1,469 & 0.398 \\
Medical specialist, mean & 152 & 117 & $<0.001$ \\
$\quad$ Salaried, \% & 49.8 & 27.6 & 0.090 \\
$\quad$ Self-employed, \% & 50.1 & 72.6 & \\
Surgeons, mean & 15 & 8 & \\
\hline
\end{tabular}

Academichospitals and private clinics were excluded to compare hospital characteristics between general hospitals with a low or high case-mix-adjusted cholecystectomy rate. ${ }^{\mathrm{a}} \mathrm{A}$ hospital was defined as "hospital with a low adjusted cholecystectomy rate" when a hospital appeared in the lowest 20th percentile of the distribution of the case-mix-adjusted cholecystectomy rates in all 3 subsequent years $\left(2013,2014\right.$, and 2015). ${ }^{\mathrm{b}}$ A hospital was defined as "hospital with a high adjusted cholecystectomy rate" when a hospital appeared in the highest 20th percentile of the distribution of the case-mix-adjusted cholecystectomy rate in all 3 subsequent years. ${ }^{\mathrm{c}}$ Involvement of surgical trainees in surgery. ure 1 . In 2014 , the 95 th/5th percentile factor score was 1.57 (top3/bottom3; 2.02). In other words, the number of case-mix-adjusted cholecystectomies per 1,000 patients was 861 in the 95th percentile and 549 in the 5 th percentile $(861 / 549=1.57)$. In 2013 and 2015, factor scores were, respectively, 1.47 (top3/bottom3; 2.03) and 1.56 (top3/ bottom3; 1.97) (Table 1).

\section{Variation between General Hospitals}

Eight academic hospitals and 1 private clinic were excluded. In 2014, the 95th/5th percentile factor scores between general hospitals was 1.28 (top3/bottom3; 1.48). The factor scores for 2013 and 2015 were 1.38 (top3/bottom3; 1.92) and 1.27 (top3/bottom3; 1.37) (Table 1).

\section{Differences between Hospitals with Low and High \\ Adjusted Cholecystectomy Rates}

Five general hospitals met the definition for a hospital with a low adjusted cholecystectomy rate (case-mix-adjusted cholecystectomy rate in the lowest 20th percentile in 3 consecutive years) and 5 general hospitals met the definition for a hospital with a high adjusted cholecystectomy rate. In hospitals with a high adjusted cholecystectomy rate, a cholecystectomy was performed earlier after diagnosis (27 vs. 47 days, $p=0.009$ ), more often by laparoscopy (98 vs. $94 \%, p<0.001$ ) and postoperative outcomes were associated with less emergency department visits within 30 days after operation ( 5 vs. $9 \%, p<0.001$ ), compared to hospitals with a low adjusted cholecystectomy rate (Table 2 ).

Hospitals with a low adjusted cholecystectomy rate were characterized by a higher percentage of a salaried medical staff, and most of the medical conditions treated in these hospitals were reimbursed by a fixed price (Table 3). Hospitals with a low adjusted cholecystectomy rate were more often teaching hospitals, with surgical trainees performing the majority of cases under supervision, while hospitals with a high adjusted cholecystectomy rate were non-teaching hospitals. Other hospital characteristics such as annual revenue, bed capacity, operation rooms, medical specialists, and surgeons were comparable between hospitals with low and high adjusted cholecystectomy rates.

\section{Discussion}

This nationwide, database study shows that hospital variation in the Netherlands in cholecystectomies is modest, cholecystectomy rates varies by $<2$-fold, and variation 
is stable over 2013-2015. This is in contrast with previous national and international data, in which cholecystectomies vary by $4-5$-fold. After excluding academic hospitals and private clinics, variation further decreased, which implies that different hospital types contribute to variation.

Previous international reports on practice variation in cholecystectomy use a different methodology, in particular to calculate factor scores; the Australian factor score for cholecystectomy is 4.1 (top3/bottom3 regional scores, Medicare insured inhabitants, adjusted for age and sex) [19] and 3.3 in the UK (highest 5/lowest 5 regional scores, inhabitants, adjusted for age and sex) [20, 21]. Several international reports only provide factor scores based on the highest/lowest; for example, a French report showed a score of 2.1 [9], and a report from the USA a score of $3.1[22,23]$. We chose not to report factor scores based on the highest/ lowest, as this methodology is way too sensitive for outliers.

One of the main explanations for the low factor score compared to other countries and conditions, beside the different methods of analyses, could be altered attitudes and beliefs about the indications for surgery between countries. During the last decade, the Dutch Society of Surgery has updated evidence-based guidelines for gallstone disease and extensively communicated these towards the practicing community and surgeons in training [3]. This certainly contributed to more uniformity in decision-making for surgery from the surgeon's perspective. Clinical trials, researching the indication for a cholecystectomy and improving the outcome after cholecystectomy, could also result in less hospital variation [24].

Although the observed variation was modest in the present study, different types of institution (academic, general, or private hospital) contributed to hospital variation. This study is the first to compare hospitals with a low and high adjusted cholecystectomy rates and showed that potentially other treatment- and hospital characteristics (e.g., time to surgery and salaried/self-employed medical staff) contribute to practice variation. Contributing factors may explain the international differences in casemix-adjusted cholecystectomy rates. Nonclinical factors contributing to practice variation may not only account for gallbladder surgery but also for other surgical procedures like total hip or knee replacements for osteoarthritis, carotid endarterectomies, coronary heart surgery, and lumbar hernia repair in which practice variation is significantly higher $[9,10,16,21]$.

Considering the patient and hospital characteristics of hospitals with low and high adjusted cholecystectomy rates, hospitals with a high adjusted cholecystectomy rate could also be smaller hospitals performing more elective, straightforward procedures. Hospitals with high adjusted cholecystectomy rates have more self-employed medical staff, which could drive surgical intervention, reduce surgical waiting lists, and receive referrals from other, larger hospitals. Surgical care in these, generally smaller, hospitals mainly focusses on less complex surgery. Patient selection in these hospitals may lead to better clinical outcomes: shorter time to surgery, higher rate of laparoscopic approach, and lower incidence of postoperative readmission. Hospitals with low adjusted cholecystectomy rates are more characterized by teaching facilities and the involvement of surgical trainees during surgery. In contrast, the absence of surgical trainees characterizes hospitals with high adjusted cholecystectomy rates. This contrast may be a reason for superior outcomes in hospitals with high adjusted cholecystectomy rates, although previous literature rejects this explanation [25-27].

It is of value that nationwide, longitudinal data were used. Additionally, practice variation data were adjusted for sex, age, and social-economic status. Moreover, secondary analyses were only performed on general hospitals, as academic hospitals and private clinics both treat a selected group of patients. Inherent to the data source, the database lacked specified clinical data on patient characteristics, comorbidity, disease prevalence, severity of disease, complicated gallbladder disease, conversion rates, and patient reported outcomes, which is a limitation of this study. Moreover, hospital variation was only measured in 3 consecutive years. Last, the majority of included patients were electively admitted via the outpatient clinic, and no differentiation could be made with the small group of patients who were admitted via the emergency department. The absence of this information may have a minor effect on the reported time interval between diagnosis and surgery.

In summary, hospital variation in cholecystectomies in the Netherlands is modest, cholecystectomy rates varies by $<2$-fold, and variation is stable over time. Different types of hospital contribute to hospital variation. Hospitals with high case-mix-adjusted cholecystectomy rates have shorter waiting lists, more self-employed staff, and their outcomes are associated with less emergency visits postoperatively.

\section{Statement of Ethics}

This research was performed in accordance with the ethical standards of the Helsinki Declaration of 1975. The Strengthening the Reporting of Observational studies in Epidemiology (STROBE) guideline and the REporting of studies Conducted using Observa- 
tional Routinely-collected health Data (RECORD) statement were followed. The present study did not require approval from an ethics committee in The Netherlands. The DRG database (with both DRGs as well as the healthcare activities) did not require written informed consent form patients.

\section{Conflict of Interest Statement}

The authors have no conflicts of interest to declare.

\section{Funding Sources}

This research was commissioned by the Dutch Patients Federation and was performed in close collaboration with the Dutch Healthcare Authority and the National Healthcare Institute. The project received funding from the Dutch Ministry of Healthcare, Welfare and Sports.

\section{Author Contributions}

Concept and design: Latenstein, Wennmacker, Groenewoud, Atsma, and de Reuver. Acquisition of data: Noordenbos. Statistical analysis: Latenstein and Noordenbos. Interpretation of data: Latenstein, Wennmacker, Groenewoud, Atsma, and de Reuver. Drafting the article: Latenstein. Critical revision of the article for important intellectual content: Wennmacker, Groenewoud, Noordenbos, Atsma, and de Reuver. All authors gave final approval of the version to be published.

\section{Data Transparency Statement}

Data, methods, and study material available: Yes. Data types: De-identified hospital data. How to access data: Philip de Reuver (Philip.dereuver@radboudumc.nl).

\section{References}

1 Russo MW, Wei JT, Thiny MT, Gangarosa LM, Brown A, Ringel Y, et al. Digestive and liver diseases statistics, 2004. Gastroenterology. 2004 May;126(5):1448-53.

2 Dutch Healthcare Authority. 2017.

3 Dutch Society of Surgery. 2016.

4 Legorreta AP, Silber JH, Costantino GN, Kobylinski RW, Zatz SL. Increased cholecystectomy rate after the introduction of laparoscopic cholecystectomy. JAMA. 1993 Sep 2229;270(12):1429-32.

5 Kang JY, Ellis C, Majeed A, Hoare J, Tinto A, Williamson RC, et al. Gallstones-an increasing problem: a study of hospital admissions in England between 1989/1990 and 1999/2000. Aliment Pharmacol Ther. 2003 Feb 15;17(4): 561-9.

6 StatLine. 2014.

7 McPherson K, Wennberg JE, Hovind OB, Clifford P. Small-area variations in the use of common surgical procedures: an international comparison of New England, England, and Norway. N Engl J Med. 1982 Nov 18;307(21): 1310-4.

8 Laycock WS, Siewers AE, Birkmeyer CM, Wennberg DE, Birkmeyer JD. Variation in the use of laparoscopic cholecystectomy for elderly patients with acute cholecystitis. Arch Surg. 2000 Apr;135(4):457-62.

9 Weeks WB, Paraponaris A, Ventelou B. Geographic variation in rates of common surgical procedures in France in 2008-2010, and comparison to the US and Britain. Health Policy. 2014 Nov;118(2):215-21.

10 Dutch Healthcare Providers. 2014.
11 Vandenbroucke JP, von Elm E, Altman DG, Gøtzsche PC, Mulrow CD, Pocock SJ, et al. Strengthening the reporting of observational studies in epidemiology (STROBE): explanation and elaboration. Epidemiology. 2014 Dec;18(6):805-35.

12 Benchimol EI, Smeeth L, Guttmann A, Harron K, Moher D, Petersen I, et al. The reporting of studies conducted using observational routinely-collected health data (RECORD) statement. PLoS Med. 2015 Oct;12(10): e1001885.

13 The Netherlands Institute for Social Research. Social Economic Status Scores. 2013, 2014, 2015.

14 Dutch Federation for Surgery. Annual Report Dutch Federation for Surgery (Dutch: Jaarverslag NVvH). 2014.

15 Dutch Ministry of Healthcare Welfare and Sports. Annual Accountability in Healthcare. 2014.

16 Australian Commission on Safety and Quality in Health Care and Australian Institute of Health and Welfare. Exploring Healthcare Variation in Australia: Analyses Resulting from an OECD Study. 2014.

17 Organisation for Economic Co-operation and Development (OECD). Geographic Variations in Health Care. 2014.

18 Wennberg JE. Time to tackle unwarranted variations in practice. BMJ. 2011;342:d1513.

19 Australian Commission on Safety and Quality in Health Care and Australian Institute of Health and Welfare. The Second Australian Atlas of Healthcare Variation: Surgical Interventions. 2014.

20 Friedman GD. Natural history of asymptomatic and symptomatic gallstones. Am J Surg. 1993 Apr;165(4):399-404.
21 Appleby J, Raleigh V, Frosini F, Bevan G, Gao H, Tom L. Variations in health care: the good, the bad and the inexplicable. London: King's Fund; 2011.

22 Perkins FM, Kehlet H. Chronic pain as an outcome of surgery: a review of predictive factors. Anesthesiology. 2000 Oct;93(4):112333.

23 Birkmeyer JD, Reames BN, McCulloch P, Carr AJ, Campbell WB, Wennberg JE. Understanding of regional variation in the use of surgery. Lancet. 2013 Sep 28;382(9898):11219.

24 van Dijk AH, Wennmacker SZ, de Reuver PR, Latenstein CSS, Buyne O, Donkervoort SC, et al. Restrictive strategy versus usual care for cholecystectomy in patients with gallstones and abdominal pain (SECURE): a multicentre, randomised, parallel-arm, non-inferiority trial. Lancet. 2019 Jun 8;393(10188):2322-30.

25 Crolla RM, van Ramshorst B, Jansen A. [Complication rate in laparoscopic cholecystectomy not different for residents in training and surgeons]. Ned Tijdschr Geneeskd. 1997 Apr 5;141(14):681-5.

26 Atta HM, Mohamed AA, Sewefy AM, AbdelFatah AS, Mohammed MM, Atiya AM. Difficult laparoscopic cholecystectomy and trainees: predictors and results in an academic teaching hospital. Gastroenterol Res Pract. 2017;2017:6467814.

27 Tafazal H, Spreadborough P, Zakai D, Shastri-Hurst N, Ayaani S, Hanif M. Laparoscopic cholecystectomy: a prospective cohort study assessing the impact of grade of operating surgeon on operative time and 30-day morbidity. Ann R Coll Surg Engl. 2018 Mar;100(3):17884. 